\title{
Endoscopic submucosal dissection using the endoscopic operation robot
}

Technological developments to minimize invasiveness are important for next-generation endoscopes; however, this should be a consistent standard during medical treatment. Thus, we have extended our focus and developed the endoscopic operation robot (EOR) to provide robotized operational support for flexible endoscopes, which can be complicated to manipulate [1]. The EOR enables distal manipulation by controlling the flexible endoscope in four axes - specifically, vertical (up-down) and horizontal angulation (left-right) using one joystick, and rotation, scope extension, and retraction using a second joystick. This report presents a further development, a unit capable of distal manipulation of insulated tip (IT) knife extension and retraction ( Fig.1), and knife tip insertion and withdrawal, which is fitted on the EOR to allow carrying out endoscopic submucosal dissection (ESD) ( $\bullet$ Fig. 2). A third joystick controls the IT-knife extension and retraction in the vertical axis as well as the insertion and withdrawal of the knife tip in the horizontal axis ( $\mathrm{Fig} .3$ ).

Three sections of a virtual lesion in a porcine resection model were resected using the EOR. As the present study focused on the specialized IT-knife unit, virtual lesion marking, localized submucosal injection of saline, initial incision, and virtual lesion recovery were carried out using conventional manual techniques. ESD was successfully achieved via distal manipulation. Excluding the manual techniques, the mean time required for EOR-mediated ESD of the three $30-\mathrm{mm}$ virtual lesion sections was 16.5 minutes.

Devices such as a multitasking platform for endoscopic full-thickness resection have been developed for natural orifice transluminal endoscopic surgery (NOTES), and it is likely that such NOTES-related devices will be developed for a peroral endoscopic approach [2]. However, the EOR is specifically designed to ensure manipulability of the flexible endoscope in the next generation of endoscope treatment. The present study indicates that the EOR can be used for ESD.

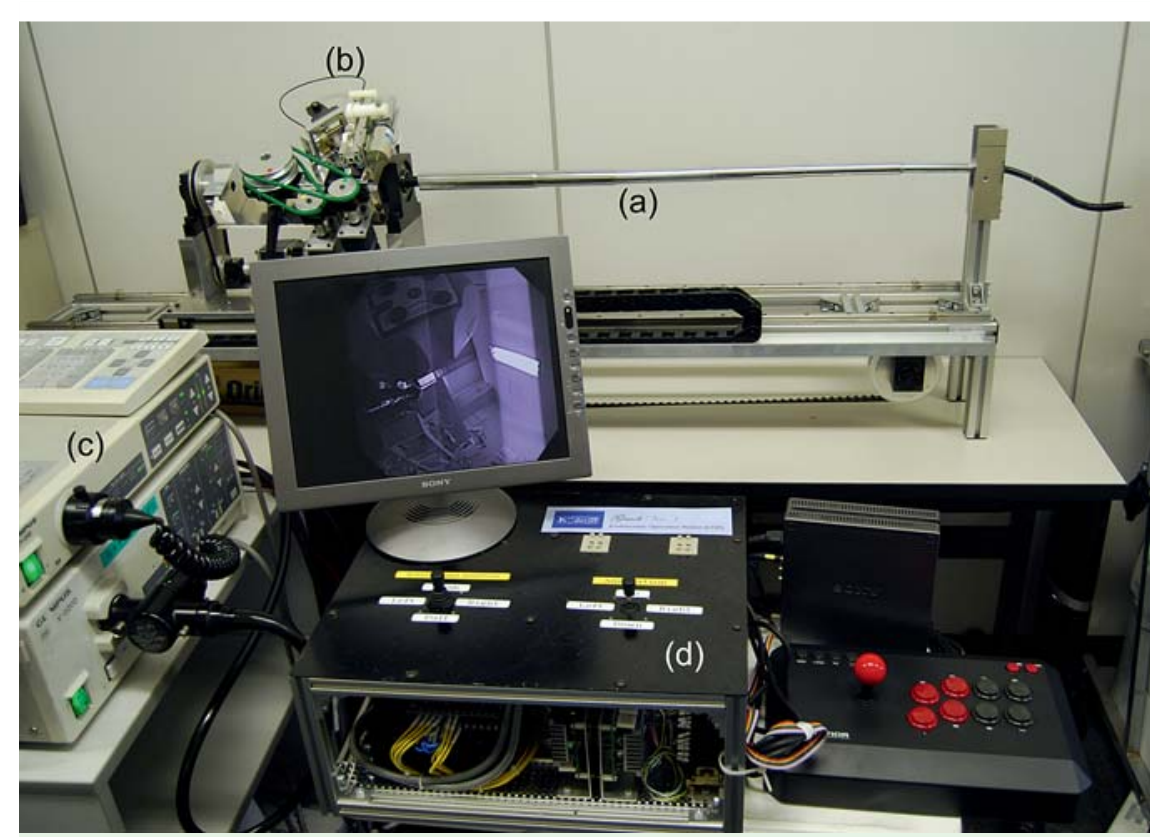

Fig. 1 The main unit (a) of the endoscopic operation robot (EOR) system with a unit capable of distal manipulation (c) of the insulated tip (IT) knife (b) and the light source unit (d).

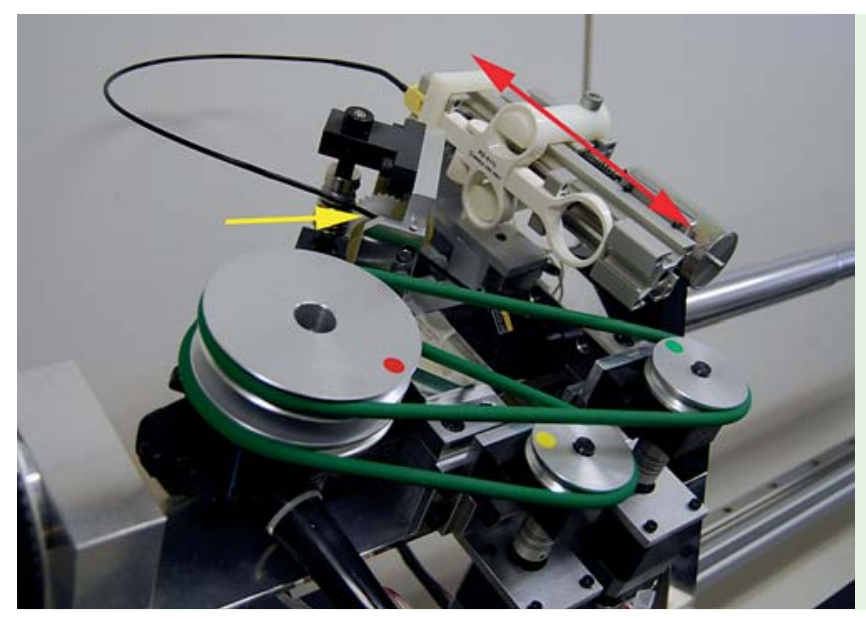

Fig. 2 Unit capable of distal manipulation of the insulated tip (IT) knife. The insertion and withdrawal of the IT knife tip is solenoid mediated (red arrow). After insertion, the IT knife is sandwiched between the rollers (yellow arrow) and motorized action is used for its extension and retraction.

\section{Acknowledgment $\nabla$}

This work was supported by Grant-in-Aid for Scientific Research (MEXT KAKENHI: 23500573).

Endoscopy_UCTN_Code_TTT_1AO_2AG

Competing interests: None

\section{K. Kume ${ }^{1}$, T. Kuroki², M. Shingai ${ }^{2}$, M. Harada ${ }^{1}$}

${ }^{1}$ Third Department of Internal Medicine, University of Occupational and Environmental Health, Japan, School of Medicine, Kitakyusyu, Japan

${ }^{2}$ Kyushu Polytechnic College, Kitakyusyu, Japan 


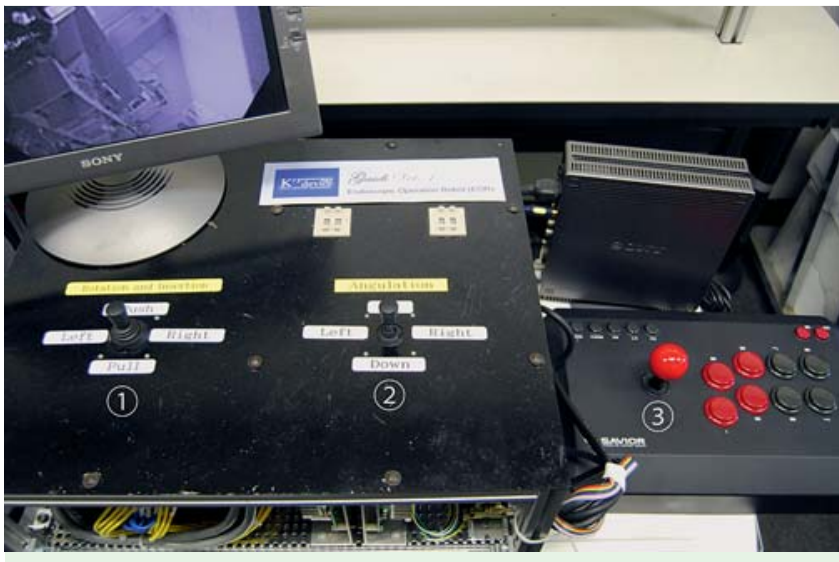

Fig. 3 The manipulation unit of the endoscopic operation robot (EOR) includes a monitor and three joysticks. The joystick on the left (1) controls tip rotation, extension, and retraction; the joystick in the center (2) controls the up - down and rightleft angulation knobs; and the joystick on the right (3) controls extension and retraction of the insulated tip (IT) knife in the vertical axis and knife tip insertion and withdrawal in the horizontal axis.

\section{References}

1 Kume K, Kuroki T, Sugihara T et al. Development of a novel endoscopic manipulation system: The Endoscopic Operation Robot (EOR). World J Gastrointest Endosc 2011; 3: $145-150$

2 Ikeda K, Sumiyama K, Tajiri $\mathrm{H}$ et al. Evaluation of a new multitasking platform for endoscopic full-thickness resection. Gastrointest Endosc 2011; 73: 117-122

\section{Bibliography}

DOI http://dx.doi.org/

10.1055/s-0032-1310251

Endoscopy 2012; 44: E399-E400

(c) Georg Thieme Verlag KG

Stuttgart · New York

ISSN 0013-726X

\section{Corresponding author}

\section{K. Kume}

K's device, Kume K. Laboratory for Endoscopy Third Department of Internal Medicine

University of Occupational and Environmental

Health

Japan

School of Medicine

1-1, Iseigaoka

Yahatanishi-ku

Kitakyusyu 807-8555

Japan

Fax: +81-93-6920107

k-kume@med.uoeh-u.ac.jp 